\title{
Performance Analysis of Communications \& Radar Coexistence in a Covert UWB OSA System
}

\author{
Shrawan C. Surender*, Ram M. Narayanan ${ }^{\dagger}$, Chita R. Das* \\ ${ }^{*}$ Department of Computer Science and Engineering, ${ }^{\dagger}$ Department of Electrical Engineering \\ The Pennsylvania State University, University Park, PA 16802, USA \\ Email: shrawans@psu.edu,ram@engr.psu.edu,das@cse.psu.edu
}

\begin{abstract}
Far-field target detection, multi-sensor communications, and security are essential requirements of first-emergency networks. A radar-communications system is a potential opportunistic spectrum access (OSA) solution, harnessing the coexisting advantages of radio detection and ranging (RADAR), and wireless communications. A multi-functional waveform has been designed, by embedding an Orthogonal Frequency Division Multiplexing (OFDM) signal within a spectrally notched ultra-wideband (UWB) random noise waveform. Extending that development, this paper analyzes the waveform's Bit-Error-Rate (BER) and Ambiguity Function (AF) formulations to demonstrate its OSA ability, that offers reliable multi-user communications, and high range and Doppler resolution in target detection. We further conclude that up to $30 \%$ of the available UWB bandwidth can be simultaneously utilized for concealed data communications without adversely affecting radar performance or its physical layer covertness.
\end{abstract}

\section{INTRODUCTION}

The capability of far-field or through-the-wall sensing of phenomenon and communicating it to a command center is a prime requirement for first-emergency networks. UWB technology, a promising avenue, not only for through-thewall radio detection and ranging (radar) [1], but also for its coexistence with wireless communications [2], is therefore, a viable solution to potentially exploit the coexisting advantages of radar and communications.

On the other hand, OFDM has gained popularity in the dynamic spectrum access arena as a prime candidate for modulation in OSA networks. Ref. [3] presents an OSA solution where the OFDM waveform as the secondary user, is sculpted to enable coexistence with a primary user. We extend this horizon towards a covert OSA solution, by working with OFDM's noise-like spectrum behavior. Our approach is to spectrally sculpt the random noise waveform of a UWB Noise Radar (the primary user), to spectrally embed OFDM based data symbols (the secondary user). This generates a covert communication platform can be formed between the individual noise radars. With this possibility to have data communication within the same spectrum as the radars transmit signal, a substantial increase in capacity and bandwidth efficiency can be achieved. The physical layer (PHY) design of such an point-to-point radar-communications system, the UWB NoiseOFDM Radar, has been analyzed in [4]. It is a hybrid radarcommunications architecture wherein both functionalities are contained, viz. UWB noise waveform for radar operation and OFDM waveform for communications operation.
Establishing a multi-user ad hoc network among such radars, can lead to data being gathered from the individual radars to be collectively processed and transformed into useful information for enhanced situational awareness. This paper, therefore, extends the UWB Noise-OFDM radar proposed in [4] to demonstrate its ability of multi-user covert communication between the radars, concurrent with the ability to perform radar operations like high-resolution target detection and ranging. The two important parameters we investigate for this performance analysis are the BER reliability measure for communications, and the AF resolution measure for radar operations [5].

Compared to generic multi-static or netted radar systems [6], the UWB Noise-OFDM radar is distinct OSA solution where the same transmit signal is simultaneously used for both radar and communication operations, and is inherently secure. In [7], a comparable dual-system is presented which uses UWB OFDM pulses for radar and communication. While this is indeed a viable alternative, immunity from detection requires random frequency hopping. Furthermore, the UWB OFDM pulses show greater range side-lobes compared to Gaussian pulses and the communication system requires full synchronization between the platforms. Further examining the existing UWB radar-communication systems, we observed that they are either not multi-radar capable [8], or are easily jammed open communication systems [7]. In contrast, we present a multifunctional UWB netted radar with better coexistence of radar and communication abilities, along with inherent transmit waveform covertness and multi-radar capability. Overall, the primary contributions of this work are:

- We demonstrate that a reliable multi-user communication platform exists in our system, which can form the physical layer for a wireless radar network. This is seen from our simulations results, which shows a BER value better than $1 \times 10^{-4}$ for an SNR of $9 \mathrm{~dB}$.

- By formulating and deriving the AF for the radar part of the Noise-OFDM Radar and comparing with the AF of a conventional Noise Radar, we show that if the fragmented gap is less than $30 \%$, then range and Doppler resolution i.e., the radar performance, is essentially unaffected.

By observing that the above two properties are due to the same composite Noise-OFDM signal, it is straight-forward to understand the coexistence of communications and radar. 


\section{BACKGROUND: SySTEM DESIGN}

The UWB Noise-OFDM Radars system architecture is briefly described in this section. Every device performs two specific functions: (1) radar specific operations such as target detection, ranging, velocity (Doppler) estimation and tracking, and (2) data-communication with other radar sensors and the centralized command center.

\section{A. Transmit Component}

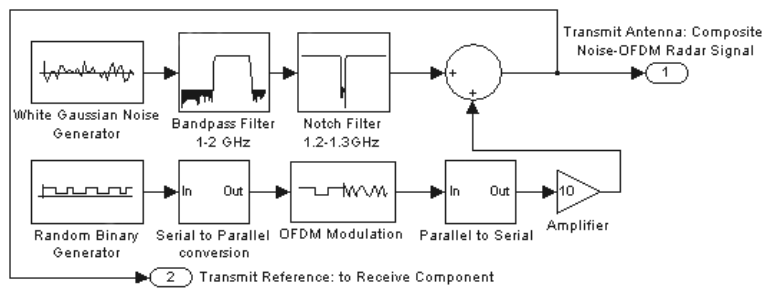

Fig. 1. UWB Noise-OFDM Radar: Transmit Component Architecture.

A noise generator produces a signal with a Gaussian amplitude distribution and approximately constant power spectral density (PSD). This signal is filtered in a Band-pass Filter (BPF) to generate a band-limited UWB noise signal within a range of $\left(f_{1}, f_{2}\right) \mathrm{GHz}$, as shown in Fig. 1. Such a bandpass Gaussian process with bandwidth $\beta$ and centered at some frequency $f_{o}$, where $f_{o}>\beta / 2$, can be expressed as

$$
X_{G}(t)=\frac{1}{2}\left[u(t) \exp \left(j 2 \pi f_{o} t\right)+u^{*}(t) \exp \left(-j 2 \pi f_{o} t\right)\right],
$$

where $u(t)=X_{c}+j X_{s}$ is the complex envelope and $*$ indicates the complex conjugation operation. In our simulations, we used a $1-2 \mathrm{GHz}$ frequency range of operation. For our system therefore, $f_{o}=1.5 \mathrm{GHz}, f_{1}=1 \mathrm{GHz}$, and $f_{2}=2 \mathrm{GHz}$. This is a UWB signal by definition since its fractional bandwidth $\left(\beta / f_{o}\right)$ is greater than 0.25 . This bandlimited noise signal is then notch-filtered within the narrower range of 1.2-1.3 GHz (in general, this could be done over any range suitable for the application). The radar system uses the full 1-2 GHz band, while the intra-sensor communications is achieved by utilizing the $1.2-1.3 \mathrm{GHz}$ sub-band. The Gaussian process $X_{N}(t)$ in the notched band, has bandwidth $\beta_{n}$ and is centered at frequency $f_{n}$, such that $f_{o}-\beta / 2<f_{n}<f_{o}+\beta / 2$. We use a random binary number generator to generate test data. In general, this would be the target data that the radar collects, or any other information it wishes to communicate to other radars in its vicinity. Because we want to hide this data within noise, and since an OFDM waveform's spectral characteristics closely mimic that of white noise, we map this data onto OFDM subcarriers to generate the OFDM symbols. A pass-band real OFDM signal with a communication carrier $f_{n}$, can be represented as [9]:

$$
X_{D}(t)=\frac{1}{2}\left[s(t) \exp \left(j 2 \pi f_{n} t\right)+s^{*}(t) \exp \left(-j 2 \pi f_{n} t\right)\right],
$$
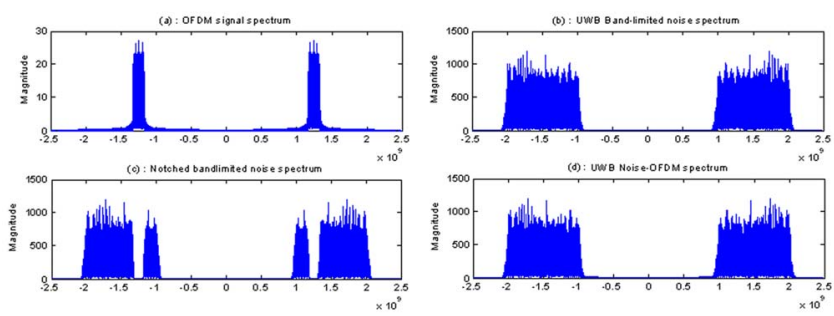

Fig. 2. Spectrum: (a) Secondary user: OFDM Signal (b) Primary user: Bandlimited UWB Noise (c) UWB Notched Noise and (d) OSA: UWB NoiseOFDM spectrum. Comparing (b) and (d), transmit waveform (d) also appears random \& noise-like, similar to (b), thereby hiding the data message.

where

$$
s(t)=\sum_{k=0}^{N-1} s_{k} \exp \left(j 2 \pi f_{k} t\right)=s_{I}(t)+j s_{Q}(t),
$$

with $s_{k}=d_{k} e^{j \theta_{k}}$ being the complex data symbols that are modulated onto the OFDM subcarriers and $f_{k}=f_{b}+k \Delta f$. Here $f_{b}$ is the base frequency, $\Delta f$ is the subcarrier spacing, and $N$ is the total number of subcarriers. These parameters are appropriately selected so that the bandwidth of this OFDM signal matches the notch bandwidth in $X_{N}(t)$.

The above OFDM signal is inserted within the notched band-limited noise signal, with appropriate power matching, to produce the multi-functional Noise-OFDM radar transmit waveform, which is propagated by an omni-directional transmit antenna. As seen by any unintended receiver, the transmitted composite waveform clearly appears random and noise-like [4], preserving its covertness properties and hence presenting physical layer security. However, for other friendly radar sensors in the transmitting radar's vicinity, the waveform is construed as data plus noise. We show four different power spectrum plots in Fig. 2(a)-2(d) to demonstrate that the transmitted signal does appear noise-like to the outside observer and its OSA behavior.

\section{B. Receive Component}

In this work, we assume a time-invariant additive white Gaussian noise (AWGN) channel, ignoring the effects of varying channel response, carrier-frequency offsets, and I/Q imbalance at the OFDM receiver. Due to the omni-directional nature of the transmit antenna and its relatively high transmit power, the Noise-OFDM data signal is likely to be picked up by several radar sensors in the neighborhood of the transmitting radar. These radar sensors process this signal within their communication-receiver-subsystem, to retrieve the concealed digital data following OFDM demodulation, decoding, and detection. The composite Noise-OFDM transmit signal also travels to the target within the radar's range and returns as target backscatter. In the transmitting radar, this echo is processed by the radar-receiver-subsystem. Herein, the echo is cross-correlated with a time-delayed replica of the transmit Noise-OFDM waveform. The correlator maximizes the output signal-to-noise ratio (SNR) and hence helps in determining the range to the target. 


\section{Physical LAyer Coexistence}

To establish coexistence of communications with radar transmissions, the receive component must be analyzed further to answer two essential issues: 1) Effect on communications performance due to the presence of UWB noise around the OFDM signal? And 2) Effect on the radar's range and Doppler estimation capabilities due to the presence of an OFDM spectrum embedded inside the UWB random noise transmit spectrum?

\section{A. Noise-embedded-communications Performance}

Bit error rate (BER) is one of the most-widely used metrics for gauging the reliability of a communication technique. For the Noise-OFDM Radar, reliability depends on various parameters: the signal-to-noise ratio $(\mathrm{SNR})$ at the input of the receiving antenna, the number of data subcarriers used, power difference between the notched noise signal and OFDM signal, and the BPF characteristics. The SNR at the input of the receiving antenna is defined as the ratio of the transmitted Noise-OFDM signal to the band-pass undesired channel noise received by the antenna. The general bit-error-rate of the Noise-OFDM Radar's communication subsystem has been derived to be [4]:

$$
P_{b}=\frac{1}{N} \sum_{l=-\frac{N}{2}}^{l=\frac{N}{2}} P_{b}\left(Y_{l}\right)=\frac{1}{N} \sum_{l=-\frac{N}{2}}^{l=\frac{N}{2}} Q\left(\sqrt{\frac{\nu_{l}^{2} \epsilon_{s, l}}{\sigma_{y, l}^{2}}}\right),
$$

where the received signal on the $l^{\text {th }}$ sub-carrier is $Y_{l}, \epsilon_{s, l}$ is the signal energy of the symbol being modulated onto the $l^{\text {th }}$ OFDM sub-carrier, $\nu_{l}$ is the FIR filter response's magnitude ripple coefficient affecting the $l^{\text {th }}$ OFDM sub-carrier and the additive white Gaussian noise has a variance $\sigma_{y, l}^{2}$ on the $l^{t h}$ OFDM sub-carrier. The perfect match of (4) with simulation results has been shown in [4]. We mention it here though, as it validates the multi-user BER vs. SNR simulations.

\section{B. Target Detection Performance}

In this sub-section, we examine the radar side of the coexisting system. The UWB noise radar is especially known for its high range resolution and good velocity resolution capabilities [10]. Range resolution states the capability of the radar to distinguish between two closely-separated targets. For a $1 \mathrm{GHz}$ UWB noise radar, this implies that targets with $15 \mathrm{~cm}$ distance between one another will be correctly detected. And velocity resolution is the radar's capability of correctly estimating the speed of different moving targets, whose velocities are closely-separated. Noting the fact that we modified the transmit waveform of a typical noise radar to embed a communication signal inside it, we investigate the UWB Noise-OFDM radar's range and velocity resolution performance by formulating its AF. For random noise radar, since its output is a random function of time, only its statistics can be described. Therefore, an analogous ambiguity function may be defined as the expected value of the response of the correlator matched to a target at a desired range moving at a desired velocity, to the return signal from the target at a different range moving at a different velocity. Consider the composite noise-OFDM signal reflected from a point target moving with constant velocity $v_{o}$ at an instantaneous range of $R=R_{o}-v_{o} t$, with resulting delay $\tau_{o}=2 R_{o} /\left(c-v_{o}\right) \cong$ $2 R_{o} / c$, and delay rate $\alpha=2 v_{o} /\left(c-v_{o}\right) \cong 2 v_{o} / c$ such that $\tau(t)=\tau_{o}-2 v_{o} t / c=\tau_{o}-\alpha t$.

For conventional radars, the transmit signal envelope is of a deterministic nature for which a matched filter can be realized, and the time-invariant ambiguity function formulation establishes its range and velocity resolution properties. However, for a random noise radar, a matched filter cannot be realized; instead a correlator matched to the transmit process is required. Since its output is a random function of time, only its statistics can be described. Therefore, an analogous ambiguity function may be defined as the expected value of the response of the correlator matched to a target at a desired range moving at a desired velocity, to the return signal from the target at a different range moving at a different velocity. To discuss the range and Doppler resolution issues pertaining to the UWB Noise-OFDM Radar, we derive its generalized wideband ambiguity function as follows.

The received target backscatter at the radar in response to

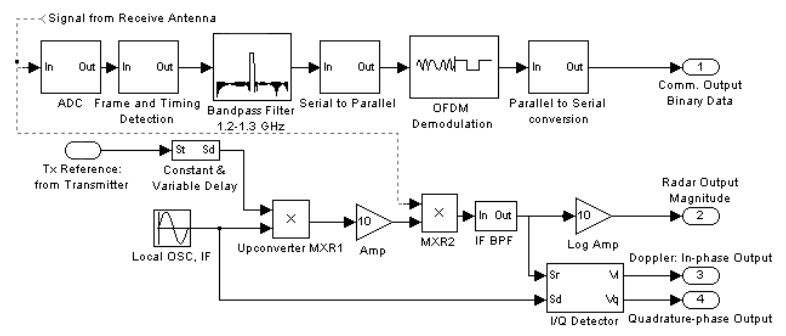

Fig. 3. UWB Noise-OFDM Radar Sensor: Receive Component Architecture.

the transmitted signal can be expressed as

$$
X_{r}(t)=k_{1} X\left((1+\alpha) t-\tau_{o}\right),
$$

where $k_{1}$ is the arbitrary amplitude constant representing target cross-section, antenna pattern, propagation factors etc.

As shown in Fig. 3, the transmitted signal is passed through a delay line with time delay equal to $\tau_{d}$ and fed into one input of an up-converter MXR1. A local oscillator at $f_{I F}$ also feeds into MXR1. Hence, the frequency shifted and time-delayed output is

$$
\begin{aligned}
X_{d}(t)= & \frac{1}{4}\left[u\left(t-\tau_{d}\right) \exp \left(j \theta_{1}\right)+u^{*}\left(t-\tau_{d}\right) \exp \left(-j \theta_{1}\right)\right] \\
& -\frac{1}{4}\left[v\left(t-\tau_{d}\right) \exp \left(j \theta_{2}\right)+v^{*}\left(t-\tau_{d}\right) \exp \left(-j \theta_{2}\right)\right] \\
& +\frac{1}{4}\left[s\left(t-\tau_{d}\right) \exp \left(j \theta_{2}\right)+s^{*}\left(t-\tau_{d}\right) \exp \left(-j \theta_{2}\right)\right],
\end{aligned}
$$

where $\theta_{1}=2 \pi\left[\left(f_{o}-f_{I F}\right) t-f_{o} \tau_{d}\right]$ and $\theta_{2}=2 \pi\left[\left(f_{n}-f_{I F}\right) t-\right.$ $\left.f_{n} \tau_{d}\right]$.

System noise, assumed to be AWGN, is distributed on the complex envelopes of the components of $X_{r}, X_{d}$ and is denoted by $\left|u_{i}, v_{i}, s_{i}\right|_{\{i=1,2\}}$. Now, passing signals $X_{r}$ and 
$X_{d}$ through mixer MXR2, filtering through the IF BPF and dropping higher-order terms, the output can be shown to be:

$$
Z_{I F}=\frac{1}{2}\left\{\operatorname{Real}\left[A_{I F}+B_{I F}\right]\right\}
$$

where

$$
\begin{gathered}
A_{I F}=\left(u_{1} u_{2}^{*}\right) \exp \left(-j 2 \pi\left(f_{I F} t+\alpha f_{o} t+f_{o}\left(\tau_{d}-\tau_{o}\right)\right)\right) \\
B_{I F}=\left(v_{1} v_{2}^{*}+s_{1} s_{2}^{*}+v_{1} s_{2}^{*}+s_{1} v_{2}^{*}\right) \\
\quad \exp \left(-j 2 \pi\left(f_{I F} t+\alpha f_{n} t+f_{n}\left(\tau_{d}-\tau_{o}\right)\right)\right) .
\end{gathered}
$$

This $Z_{I F}$ is then passed through the I/Q integrating filters with impulse response $h(t)$. The filter impulse response $h(t)$ could be of any type; however, in this analysis, we assume a rectangular time window for the filter response of the form

$$
h(t)=1 / T, \text { for } 0 \leq t \leq T ; 0 \text {, otherwise. }
$$

To estimate $Z_{I F}$, we evaluate $u_{1} u_{2}^{*}, v_{1} v_{2}^{*}, s_{1} s_{2}^{*}, v_{1} s_{2}^{*}$ and $s_{1} v_{2}^{*}$ individually and take the expectation, which results in:

$$
\begin{aligned}
& E\left\{u_{1} u_{2}^{*}\right\}=\left\{R_{c c}(\alpha t-\tau)-j R_{c s}(\alpha t-\tau)\right\} \\
& E\left\{v_{1} v_{2}^{*}\right\}=\left\{R_{c c_{n}}(\alpha t-\tau)-j R_{c s_{n}}(\alpha t-\tau)\right\} \\
& E\left\{v_{1} s_{2}^{*}\right\}=E\left\{s_{1} v_{2}^{*}\right\}=0
\end{aligned}
$$

where

$$
R_{c c}(.)=\frac{\sin [\pi \beta(\alpha t-\tau)]}{\pi \beta(\alpha t-\tau)} ; \quad R_{c c_{n}}(.)=\frac{\sin \left[\pi \beta_{n}(\alpha t-\tau)\right]}{\pi \beta_{n}(\alpha t-\tau)}
$$

are the system auto-correlation functions.

For evaluating $s_{1} s_{2}^{*}$, we reasonably assume that identical data are transmitted over all sub-carriers of the OFDM symbol. We then use the orthogonality principle for OFDM carriers and equation (3) together, to finally show that $E\left\{s_{1} s_{2}^{*}\right\}=N T_{s}$, where $T_{s}$ is the symbol period.

Neglecting the phase terms, the expected values of the I and $\mathrm{Q}$ outputs of the $\mathrm{I} / \mathrm{Q}$ integrating filters can therefore be symbolized as $\left\langle Z_{I}(\alpha, \tau, t)\right\rangle$ and $\left\langle Z_{Q}(\alpha, \tau, t)\right\rangle$.

Since the envelope of the output is defined to be the RMS sum of these two, the ambiguity function is of the form:

$$
|\langle\chi(\alpha, \tau, t)\rangle|=\left|\left\langle Z_{I}\right\rangle^{2}+\left\langle Z_{Q}\right\rangle^{2}\right|^{1 / 2} .
$$

Similar to [10], we go through the algebra of finding $\left\langle Z_{I}\right\rangle$ and $\left\langle Z_{Q}\right\rangle$, squaring and summing them, and use trigonometric identities to solve for equation (11). Ignoring the crosscorrelation component due to the UWB noise as well as the notch spectrum (which is very low, hence negligible for a more uniform transmit PSD), equation (11) then reduces to the following simplified expression, which is the ambiguity function of a UWB Noise-OFDM Radar:

$$
|\langle\chi(\alpha, \tau, t)\rangle|=|(A+B) \otimes h(t)|,
$$

where

$$
\begin{aligned}
& A=R_{c c}(\alpha t-\tau) \exp \left(-j 2 \pi \alpha f_{o} t\right) \\
& B=\left(R_{c c_{n}}(\alpha t-\tau)+N T_{s}\right) \exp \left(-j 2 \pi \alpha f_{n} t\right) .
\end{aligned}
$$

The significance of this result, as drawn from our simulations in Section IV, is that the OFDM data bandwidth can be around 30\% without adversely affecting ranging or Doppler estimation performance, noise-like properties of the radar.

\section{Simulation Results}

To validate the concepts mentioned above, we used MATLAB as the simulation platform. With $f_{1}, f_{2}$ and $f_{n}$ the same, a 253-tap finite-impulse-response (FIR) equiripple band-pass filter with a transition region bandwidth of $\approx 50 \mathrm{MHz}$ and a pass-band magnitude ripple of $\pm 0.5 \mathrm{~dB}$ was used in the receiver. The input parameters were the number of subcarriers being used (which was 32 to cover a $100 \mathrm{MHz}$ notch), the number of symbols per carrier (50 in our case), the bin length (1024), sample frequency (5 GHz) and the channel SNR. Based on this required channel SNR, appropriate noise power was added to the transmitted signal.

\section{A. Multi-user Radar-Communications Performance}

To study the multi-user BER vs. SNR characteristics of the Noise-OFDM radar, consider a multi-radar system where a radar $i$ has neighbors $j$ and $k$. We assume that $i$ is allowed to communicate with $j$ on the odd-numbered subcarriers, and with $k$ on the even-numbered subcarriers respectively. Both $j$ and $k$ individually produce their transmit NoiseOFDM waveform and transmit it to $i$. Upon receiving their transmissions as a composite AWGN-affected waveform, $i$ demodulates the incoming signal and separates $j$ and $k$ 's data bits. Fig. 4 shows the BER vs. SNR performance. Comparing Noise-OFDM to a pure OFDM signal, we observe that multiuser presence does quite heavily affect the noise-OFDM system, as can be seen from its high error-floor. However, utilizing a 3-carrier data redundancy technique (Noise-OFDM with data redundancy (DR)) as in [4], we see that this floor can be completely removed and the performance remarkably improves. Our system is able to achieve a BER better than $1 \times 10^{-4}$ for an SNR of $9 \mathrm{~dB}$. For this same BER, there is still an 8-dB difference though, from pure OFDM's performance. It is preferable to maintain this gap, as it is the price that we pay to achieve the PHY security that this system presents [4] to the transmit waveform.

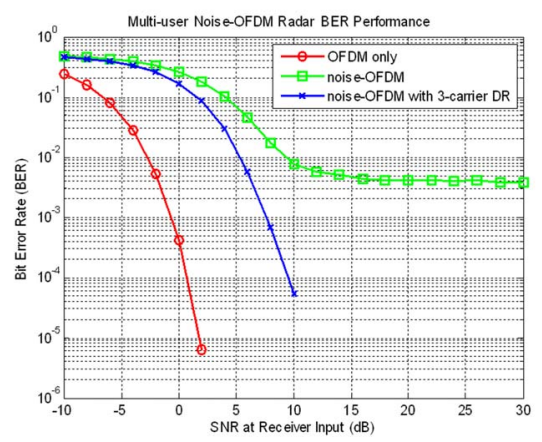

Fig. 4. Noise-OFDM radar multi-user performance in AWGN channel.

\section{B. Radar Operations}

Target ranging, Doppler estimation, range resolution and Doppler resolution are the important metrics for measuring the performance of UWB noise radar. It was already shown in [4], that the range estimation performance of the UWB 


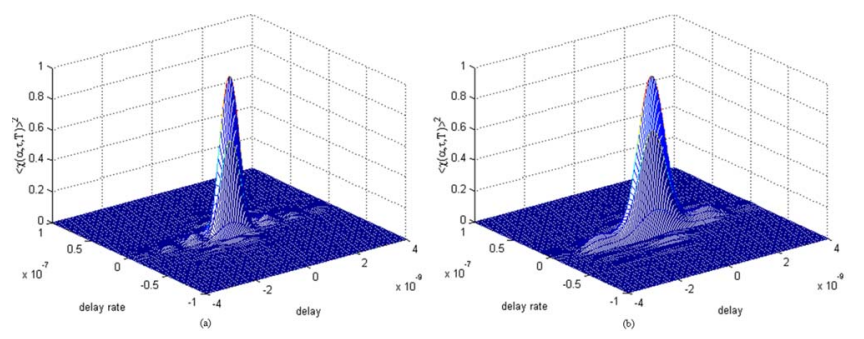

Fig. 5. AF for a (a) UWB Noise Radar of 1 to $2 \mathrm{GHz}$, [10], (b) Noise-OFDM Radar with $300 \mathrm{MHz}$ notch bandwidth.

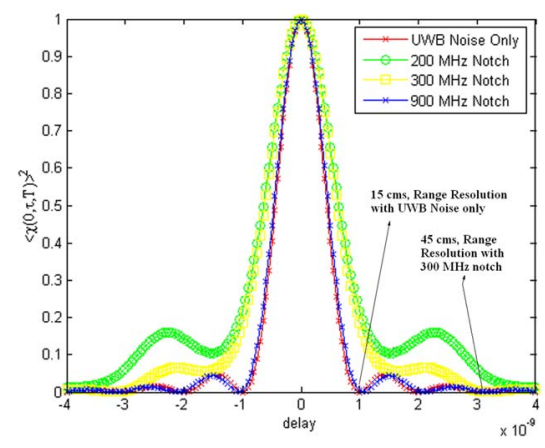

Fig. 6. For $\alpha=0$, the 2-D AF plot showing range resolution.

Noise-OFDM radar is unaffected by the presence of an OFDM signal in the transmit waveform. In this paper, for further verifying radar performance in the OSA presence of embedded communications, we simulated the AF expressed in equation (12). From the AF plot, range and Doppler resolution can be inferred, since range resolution $\Delta R=c T / 2$, where $c=3 \times$ $10^{8} \mathrm{~m} / \mathrm{s}$, and $T=1 /$ bandwidth is the first zero-crossing of the AF along $\tau$ axis. We used an integration time of $50 \mathrm{~ms}$, a notch bandwidth of $300 \mathrm{MHz}$, and a center frequency of $1.45 \mathrm{GHz}$. The squared outcome (reduces side-lobes) of the convolution in equation (8) at time $t=T$ was stored for each $\alpha$ and $\tau$. The resulting $\mathrm{AF}$ and its slice along $\tau$ axis is plotted in Fig. 5-(b) and Fig. 6, respectively. From Fig. 5-(b), it can be noted that the main lobe width (proportional to range resolution) remains relatively same as compared to a conventional UWB Noise Radar's AF main lobe in Fig. 5-(a) [10], although its side lobes are slightly higher. Fig. 6 in further detail, compares the range resolution of a UWB Noise Radar with a UWB Noise-OFDM Radar of varying notch bandwidths. Since our OSA goal is to have concealed coexistence of data with radar, we observe that a reasonable tradeoff can be reached between efficient wireless communications and covert radar operations: for e.g. as seen in Fig. 6, $300 \mathrm{MHz}(30 \%)$ communications bandwidth is available with $45 \mathrm{~cm}$ radar range resolution in the UWB Noise-OFDM radar compared to $15 \mathrm{~cm}$ range resolution of conventional UWB Noise radar with no communications ability. It is also seen that range resolution gets better as the notch bandwidth (embedded OFDM bandwidth) is increased i.e., $15 \mathrm{~cm}$ for $900 \mathrm{MHz}$ notch. However, as the embedded OFDM bandwidth increases, the overall noise-like behavior of the signal decreases [4], hence reducing its covertness.
Furthermore, a slice of Fig. 5-(b) along the $\alpha$ axis, showed that the Doppler resolution inferred as above, but along the $\alpha$ axis, does not change with the varying sizes of the notch bandwidth. This is as expected since the velocity resolution only depends on the integration time [10] and not on the waveform bandwidth. Therefore, with a $30 \%$ notch we are able to maintain the UWB noise radar's covertness and target resolution, while simultaneously using a portion of its bandwidth for communication.

\section{CONCLUSions}

In this work, we present a unique covert opportunistic spectrum access solution to enable the coexistence of OFDM-based data communications with UWB noise radars (the primary user). Using the same multi-functional composite waveform, we have demonstrated that reliable multi-user data transmission/reception can occur simultaneously with high resolution target detection/ranging. Towards this we also formulated the ambiguity function of this multi-functional waveform and have shown that around $30 \%$ of a $1 \mathrm{GHz}$ UWB bandwidth is available for data communications, without adversely affecting the primary function of the radar. Promising and prospective candidates for implementing this system are Software Defined Radio (SDR) techniques of software-based signal processing, hardware such as the Universal Software Radio Peripheral (USRP) and high-speed digital-to-analog (DAC) converters. This will allow the system parameters to be software configurable, hence making an effective solution to dynamic spectrum access in first-emergency networks.

\section{REFERENCES}

[1] C. Gentile and A. Kik, "WLC28-4: An evaluation of ultra wideband technology for indoor ranging," in Proc. of GLOBECOM 06, San Francisco, CA, USA, doi: 10.1109/GLOCOM.2006.778, Dec. 2006.

[2] J. Bellorado, S. S. Ghassemzadeh, L. J. Greenstein, T. Sveinsson, and V. Tarokh, "Coexistence of ultra-wideband systems with IEEE-802.11a wireless lans," in Proc. of GLOBECOM 03, vol. 1, San Francisco, CA, USA, pp. 410-414, December 2003.

[3] Z. Wang, D. Qu, T. Jiang, and Y. He, "Spectral sculpting for OFDM based opportunistic spectrum access by extended active interference cancellation," in Proc. of GLOBECOM 08, New Orleans, LA, USA, doi: 10.1109/GLOCOM.2008.ECP.852, Dec. 2008.

[4] S. C. Surender and R. M. Narayanan, "UWB Noise-OFDM netted radar: Physical layer design and analysis," IEEE Transactions on Aerospace and Electronic Systems, Accepted Dec. 2009, In Press.

[5] K. J. Quirk and M. Srinivasan, "An MSK waveform for radar applications," in Proc. of GLOBECOM 09, Honolulu, Hawii, USA, doi: 10.1109/GLOCOM.2009.5425647, Dec. 2009.

[6] C. J. Baker and A. L. Hume, "Netted radar sensing," IEEE Aerospace and Electronic Systems Magazine, Vol. 3, Issue 2, pp. 3-6, Feb. 2003.

[7] D. Garmatyuk, J. Schuerger, Y. T. Morton, K. Binns, M. Durbin, and J. Kimani, "Feasibility study of a multi-carrier dual-use imaging radar and communication system," in Proc. of the European Microwave Conference (EuMC 2007), Munich, Germany, pp. 1473-1476, October 2007.

[8] G. N. Saddik, R. S. Singh, and E. R. Brown, "Ultra-wideband multifunctional communications/radar system," IEEE Transactions on Microwave Theory and Techniques, vol. 55, no. 7, pp. 1431-1437, July 2007.

[9] Y. Li, Orthogonal Frequency Division Multiplexing for Wireless Communications. New York, NY: Springer, 2006.

[10] M. Dawood and R. M. Narayanan, "Generalised wideband ambiguity function of a coherent ultrawideband random noise radar," IEE Proceedings on Radar, Sonar and Navigation, vol. 150, no. 5, pp. 379 - 386, October 2003. 